\title{
Histological study of chronic pulmonary aspergillosis
}

\author{
Naobumi Tochigi ${ }^{1 \dagger}$, Takao Ishiwatari ${ }^{1 \dagger}$, Yoichiro Okubo ${ }^{1}$, Tsunehiro Ando ${ }^{1}$, Minoru Shinozaki ${ }^{1}$, Kyoko Aki $^{1}$, \\ Kyoko Gocho ${ }^{2}$, Yoshinobu Hata ${ }^{3}$, Somay Y. Murayama ${ }^{4}$, Megumi Wakayama ${ }^{1}$, Tetsuo Nemoto ${ }^{1}$, Yasuhiro Hori ${ }^{1}$ \\ and Kazutoshi Shibuya ${ }^{1 *}$
}

\begin{abstract}
Background: Chronic pulmonary aspergillosis (CPA) has been accepted the criteria for the diagnosis of pulmonary Aspergillus infection. Whereas, either pathophysiology or signs of CPA remains still controversial.

Methods: In this study, we histopathologically investigated 25 specimens of CPA, surgically resected.

Results: 21 (84\%) of that comprised male. There were 21 cases with mild impairment of the immune system and/or a scar mostly due to old tuberculosis. There is a tendency for a negative correlation between peripheral blood white cell numbers and value level of beta-(1,3)-D-glucan. Four cases showed a granular fluorescent signal in granulation tissue surrounding the cavity without the fungal aspects itself.

Conclusions: In conclusion, acute inflammatory exudate along the terminal respiratory tract is most significant pathophysiolocial complication of the CPA, caused to organizing pneumonia, which derives fatal respiratory failure. In addition, the viability of fungus does not concern extension of exudative inflammation at the site of erosion along terminal airway.
\end{abstract}

\section{Background}

Chronic pulmonary aspergillosis (CPA) has become an accepted criterion for the diagnosis of pulmonary Aspergillus infection, whereas the use of other aspects involving pathophysiology or clinico-pathology remains controversial. CPA tends to occur in elderly and/or debilitated individuals who might not otherwise be immunodeficient. The underlying chronic cavitary lung disease may be due to prior tuberculosis, bullous lung disease, chronic interstitial disease, lung irradiation, surgical lung resection, lung infarction, or cystic fibrosis [1]. End-stage sarcoidosis is a common cause of the cystic remodel associated with CPA. Pathophysiology of CPA may be essentially defined by epithelial destruction and localized infiltration of fungi induced by mild impairment of the immune system with airway anatomical reconstruction. Previously, semi-invasive pulmonary aspergillosis [2], chronic necrotizing pulmonary aspergillosis [3], and chronic cavitary pulmonary

\footnotetext{
*Correspondence: kaz@med.toho-u.ac.jp

${ }^{\dagger}$ Equal contributors

'Department of Surgical Pathology, Toho University School of Medicine,

6-11-1 Omori-nishi, Ota-ku, Tokyo 143-8541, Japan

Full list of author information is available at the end of the article
}

aspergillosis [4] were suggested clinically. However, there are few histopathological studies of CPA [5]. In this study, we investigated surgically resected CPA specimens histopathologically and analyzed the structure of the preexisting cavity found in most cases. Additionally, we attempted to clarify the pathogenesis of CPA by analyzing laboratory data from CPA cases, and suggest effective tools to monitor the pathogenesis of CPA.

\section{Materials and methods}

This study was approved by the ethics committee of Toho University (approval number: 2600524051). We reviewed the medical records of Toho University Omori Medical Center from 1999 to 2013, and found 25 surgically resected CPA cases. Firstly, we analyzed the character of the cavity surrounding the fungus ball histopathologically using hematoxylin-eosin double stain (H-E), Grocott's methenamine silver stain (GMS), and elastic van Gieson stain (EVG). Immunohistochemical staining for cytokeratin was done as a routine procedure. We measured the erosion ratio for each case using a representative slide. Secondly, we collected laboratory data including white blood cells in peripheral blood (WBC), c-reactive protein 
(CRP), and beta-(1,3)-D-glucan (BD). In the BD assay, we used Fungitec ${ }^{\circ} \mathrm{G}$ test MK (Seikagaku Corporation, Tokyo) or MK-II (Nissui Pharmaceutical Co. Ltd., Tokyo). Thirdly, we analyzed the diffusion of fungal ingredient which can be detected as fine granules by glucan-specific tissue fluorescent assay system (Fungiflora $Y^{\oplus}$, Trust Medical Co. Ltd., Kasai, Hyogo) at the eroded tissue consisting cavity wall.

\section{Results}

Table 1 summarizes details of the 25 surgically resected CPA cases. The age of patients varied from 28 to 78 years (median 60), 21 cases (84\%) were male, and all cases involved an upper lobe lesion. There was no severe impairment of the immune system, such as malignant hematopoietic tumor or cytotoxic chemotherapy. However, mild impairment of the immune system occurred by a low dose of cortico-steroid administration
(SA) and/or diabetes mellitus (DM). Additionally, a scar mostly due to old tuberculosis (OT) was recorded in nine cases. There were 21 cases (84 \%) with SA and/or DM and/or OT.

Histopathological examination revealed erosion of the cavity surrounding the fungus ball in all 25 cases. Nine cases exhibited eosinophil infiltration clearly. No epithelioid cell granuloma was detected. The erosion ratio was 3.7-100 \% (mean: $62.0 \%$ ). The mean erosion ratio with the Splendore-Hoeppli phenomenon $(\mathrm{SH})$ was $53.2 \%$, otherwise it was $71.4 \%$ without SH (Fig. 1). Interestingly, there was a wide organization area surrounding the cavity without fungal aspects in some cases (Fig. 2). This suggests that we cannot explain this phenomenon using conventional infectious theory which involves local proliferation of microorganisms.

A correlation between WBC and BD is shown in Fig. 3. There is a tendency for a negative correlation between WBC and BD, although it was not statistically

Table 1 Character of 25 surgically resected CPA cases

\begin{tabular}{|c|c|c|c|c|c|c|c|c|c|c|c|c|c|}
\hline Age & Sex & Locus & SA & DM & OT & CRP & WBC & $B D$ & ER & $\mathrm{SH}$ & El & $\mathrm{CO}$ & GF \\
\hline 28 & $M$ & $R U$ & & & & 0.2 & 7.1 & 12.3 & 78.3 & & & & \\
\hline 37 & $M$ & $\mathrm{RU}$ & $Y$ & & & 0.4 & 5.4 & N/A & 100.0 & & & & \\
\hline 39 & $F$ & LU & Y & & & 15.5 & 14.0 & 11.0 & 100.0 & & & $Y$ & $Y$ \\
\hline 43 & $F$ & LU & & & & 2.3 & 5.7 & N/A & 100.0 & & & & \\
\hline 43 & $M$ & $\mathrm{RU}$ & & & $Y$ & 7.9 & 7.6 & 9.3 & 32.7 & & $Y$ & & \\
\hline 43 & $M$ & LU & $Y$ & & & 0.3 & 11.8 & 23.9 & 11.4 & $Y$ & & & \\
\hline 50 & $M$ & $\mathrm{RU}$ & & Y & & 0.1 & 10.6 & 5.0 & 100.0 & Y & $Y$ & & \\
\hline 55 & $M$ & LU & & $Y$ & $Y$ & 0.5 & 5.7 & 492.0 & 46.7 & $Y$ & & & Y \\
\hline 56 & $F$ & $\mathrm{RU}$ & & & Y & 0.2 & 4.9 & 8.7 & 46.2 & $Y$ & & & \\
\hline 57 & $M$ & RU & & $Y$ & $Y$ & 1.0 & 5.8 & $\mathrm{~N} / \mathrm{A}$ & 42.9 & $Y$ & $Y$ & & \\
\hline 58 & $M$ & LU & & & Y & 0.2 & 3.1 & 67.6 & 44.7 & Y & & Y & $Y$ \\
\hline 59 & $M$ & RU & & Y & & 0.2 & 13.5 & N/A & 100.0 & & Y & & \\
\hline 60 & $M$ & RU & $Y$ & & & 0.7 & 10.7 & 5.0 & 57.9 & & & & \\
\hline 61 & $M$ & $R U$ & Y & Y & & 0.3 & 9.9 & 27.7 & 54.5 & $Y$ & & & \\
\hline 61 & $M$ & LU & & & & 0.6 & 6.2 & 6.1 & 3.7 & $Y$ & Y & & \\
\hline 64 & $M$ & $\mathrm{RU}$ & & & Y & 0.1 & 5.1 & 18.2 & 38.7 & & & & \\
\hline 66 & $M$ & LU & & & & 6.6 & 9.3 & 5.0 & 75.5 & Y & Y & & \\
\hline 67 & $F$ & LU & & & Y & 1.4 & 6.7 & 24.2 & 79.3 & $Y$ & & & \\
\hline 68 & $M$ & $\mathrm{RU}$ & & $Y$ & & 0.7 & 9.3 & 5.0 & 81.1 & & $Y$ & $Y$ & \\
\hline 69 & $M$ & LU & $Y$ & & & 7.9 & 6.5 & 11.4 & 3.8 & & & & \\
\hline 71 & $M$ & $\mathrm{RU}$ & & $Y$ & $Y$ & 2.0 & 6.7 & 5.2 & 85.4 & Y & $Y$ & & \\
\hline 73 & $M$ & $\mathrm{RU}$ & & & & 5.4 & 8.2 & N/A & 88.6 & $Y$ & & $Y$ & $Y$ \\
\hline 74 & $M$ & $\mathrm{RU}$ & & $Y$ & & 0.2 & 4.0 & 29.8 & 95.3 & & & & \\
\hline 75 & $M$ & LU & & $Y$ & $Y$ & 1.9 & 4.2 & 7.1 & 12.9 & $Y$ & & & \\
\hline 78 & $M$ & RU & & $Y$ & & 4.8 & 7.5 & 10.7 & 69.2 & & $Y$ & & \\
\hline
\end{tabular}

$R U$ right upper lobe, $L U$ left upper lobe, $S A$ history of cortico-steroid administration, $D M$ diabetes mellitus, OT scar mostly due to old tuberculosis, CRP c-reactive protein, WBC white blood cells in peripheral blood, $B D$ beta-(1, 3)-D-glucan, ER erosion ratio (\%), SH Splendore-Hoeppli phenomenon, El eosinophil infiltration, $\mathrm{CO}$ calcium oxalate crystal deposition, GF granular fluorescent signal at granulation tissue surround the cavity, but not identified fugal aspects 


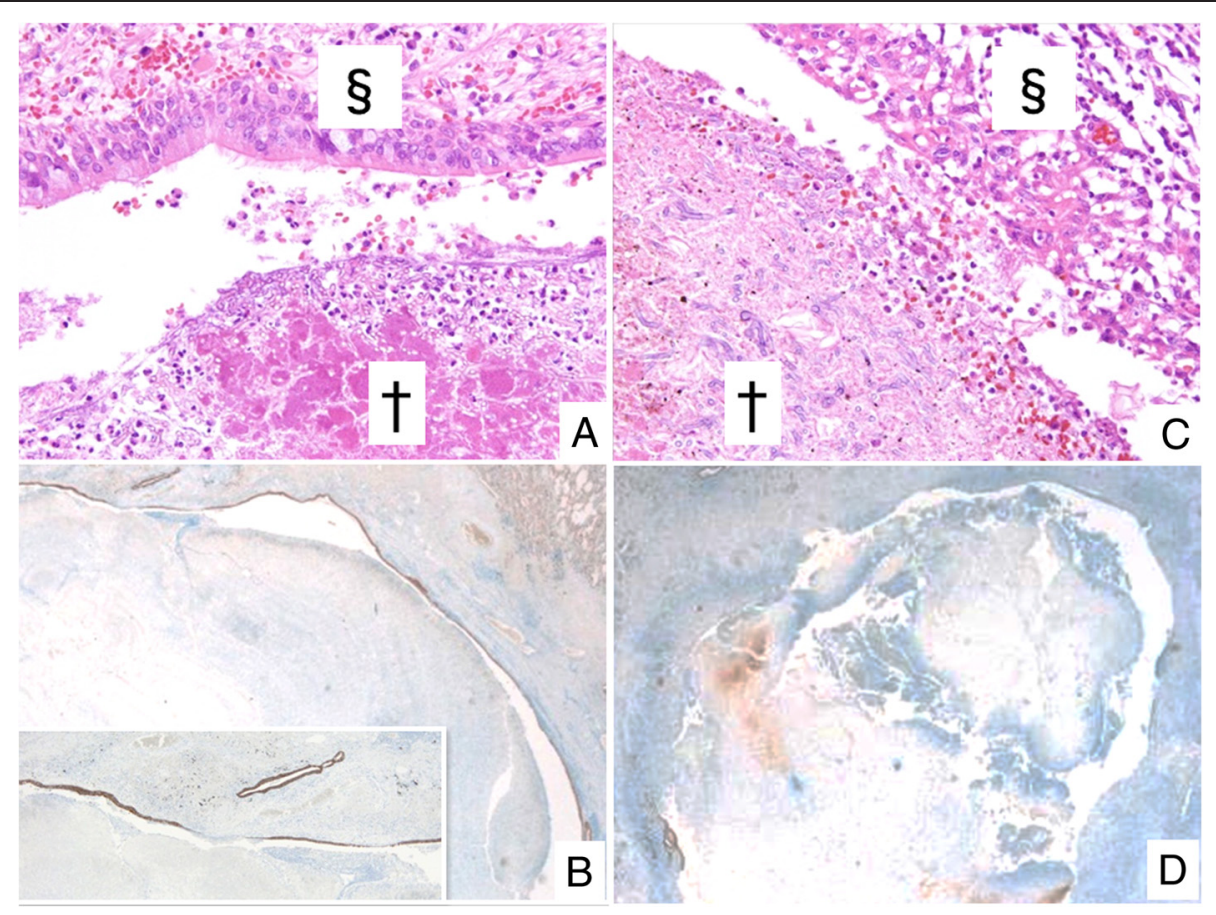

Fig. 1 Histopathology of erosion caused by CPA. a Cavity [§] is covered by ciliated epithelium without erosion (H-E). Note the Splendore-Hoeppli phenomenon (eosinophilic staining in fungus ball [†]). $\mathbf{b}$ Immounohistochemical staining for cytokeratin AE1/AE3 is done in case A. Erosion ratio is $3.7 \%$. c Epitheliums disappear and inflammatory granulation tissue exposes the surface of cavity. $\mathbf{d}$ Immounohistochemical staining for cytokeratin AE1/AE3 is done in case A. Erosion ratio is $95.3 \%$
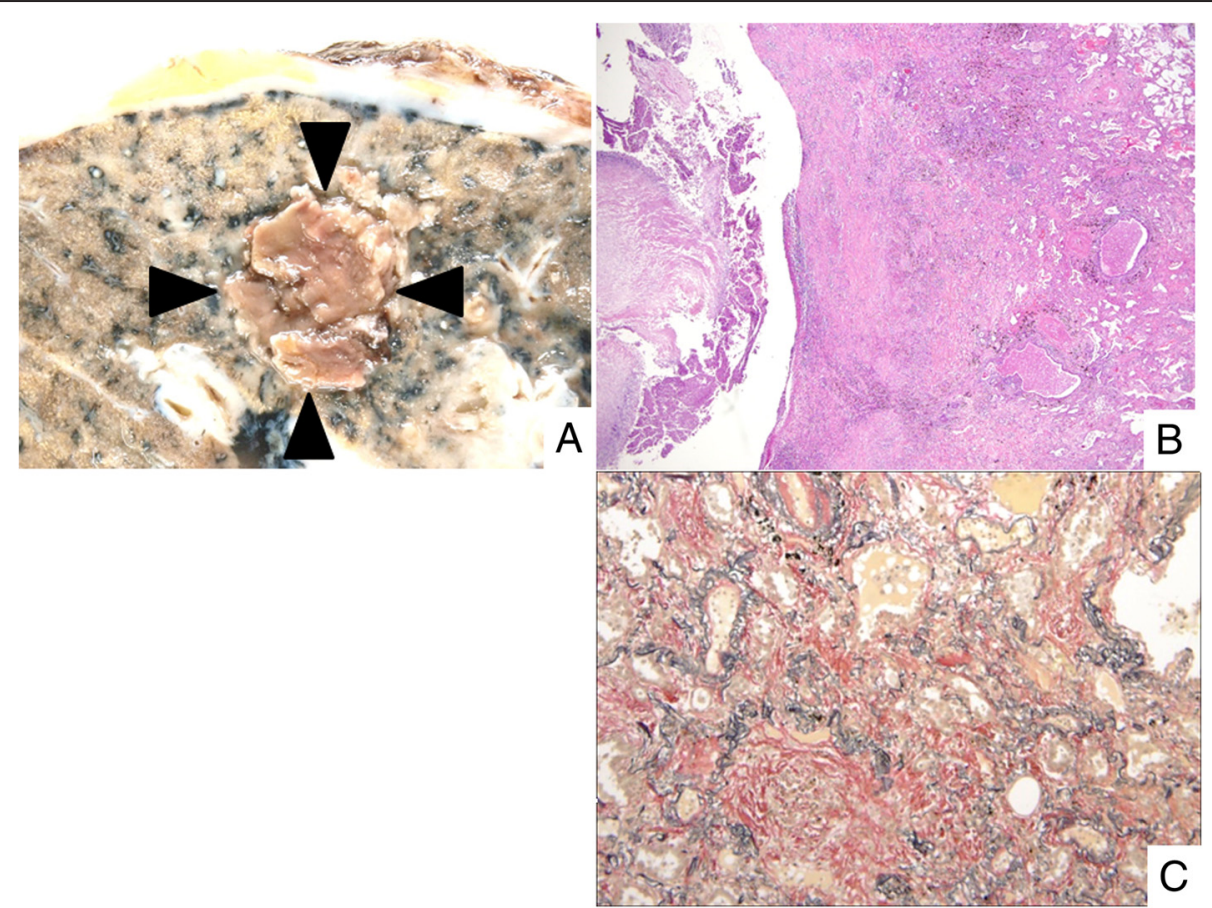

Fig. 2 Histopathology of organization surrounding the cavity. a Macroscopic findings reveal the fungus ball (arrow head). Pleura with fibrous thickening is also noted. b Organization area can be seen around the fungus ball (H-E staining). c Alveolar spaces are filled with dense collagenous tissue (EVG staining) 


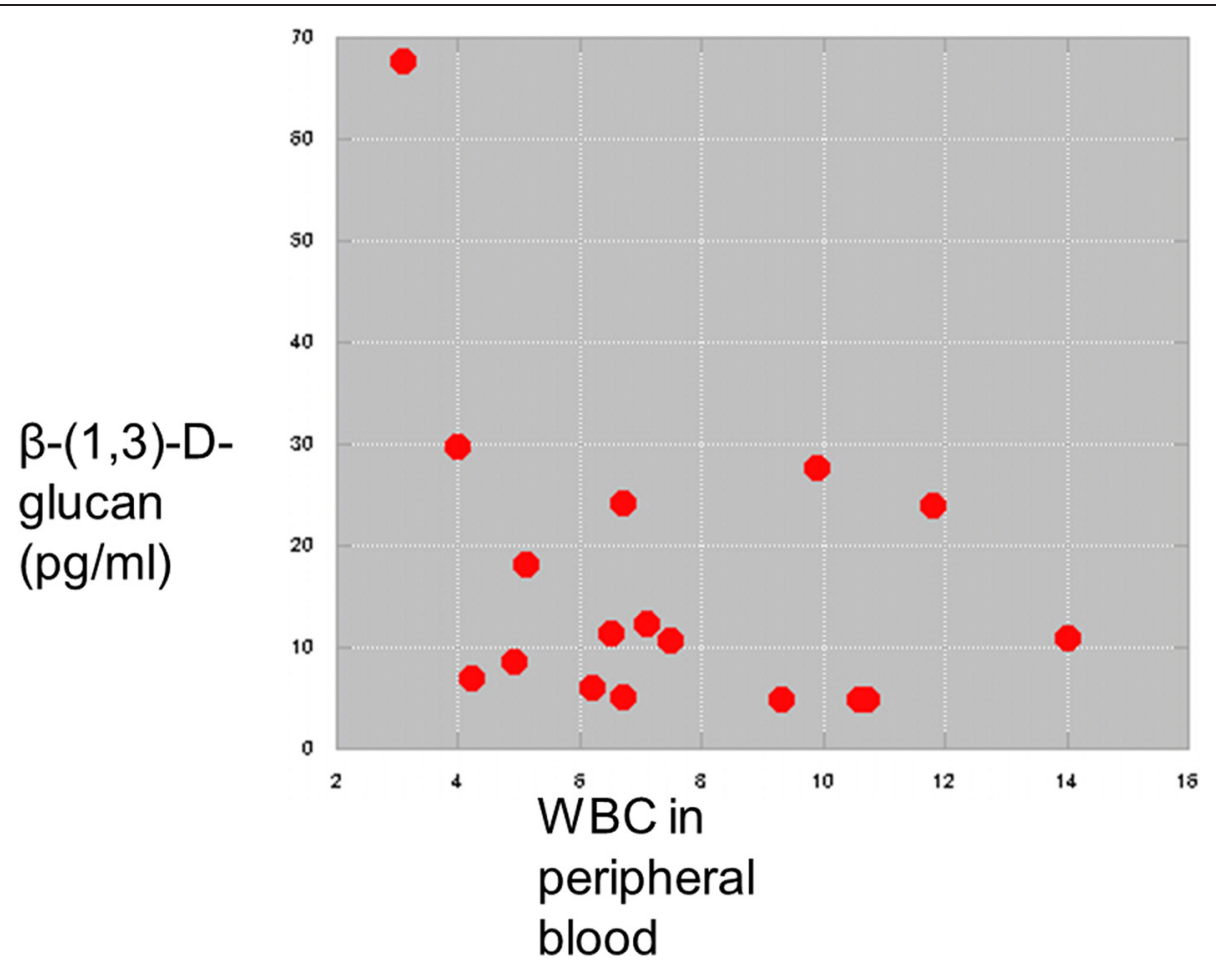

Fig. 3 Correlations between WBC and BD

significant. These data suggest that impairment of the immune system worsens CPA pathogenesis.

Four cases (16\%) showed a granular fluorescent signal in granulation tissue surrounding the cavity using Fungiflora $\mathrm{Y}^{\ominus}$ stain, but we did not identify fungal aspects (GF) using H-E or GMS (Fig. 4). Among the 19 cases with measured $\mathrm{BD}$ before the surgical resection, the three GF-positive cases had a BD of $190.2 \pm 201.2 \mathrm{pg} / \mathrm{ml}$, while the $16 \mathrm{GF}-$ negative cases had a BD of $12.8 \pm 7.5 \mathrm{pg} / \mathrm{ml}$. The detected correlation between the presence of GF and the high level of BD is statistically significant (one-sided test, $p=0.027$, Mann-Whitney $U$ test).

\section{Discussion}

Gefter et al. defined semi-invasive pulmonary aspergillosis as a chronic cavitary form of pulmonary aspergillosis with mild immunosuppression or underlying lung disease [2]. They indicated that this form of aspergillosis was part of a spectrum of disease ranging from saprophytic to frankly invasive types. This was followed by report from Binder et al. which finally defined chronic necrotizing pulmonary aspergillosis [3]. Within recent decade, Denning et al. described chronic cavitary pulmonary aspergillosis and chronic fibrosing pulmonary aspergillosis using radiological findings [4]. On the other hand, because of the difficulty to distinguish chronic necrotizing pulmonary aspergillosis and chronic cavitary pulmonary aspergillosis,
Izumikawa et al. proposed chronic progressive pulmonary aspergillosis, which included chronic pulmonary aspergillosis of both type; necrotizing and cavitation [6]. However, there are few reports conducting histological and pathophysiological analyses on this chronic form of aspergillosis with reference to some representative monitoring system. Whereas our literature search for previous study could highlight the best-constructed histopathological study of Yousem reviewed $10 \mathrm{CPA}$ cases and yielded a classification with three categories of granulomatous response [5], little were discussed on the pathophysiology of the disease. With comparison to Yousem's subject group, there might be closer relationship between sequela of tuberculosis and CPA emerged from our study which can be supported by a result that our 25 CPA cases involved an upper lobe that the commonest area of the primary lesion of tuberculosis. However, since none of our patient showed active granuloma that must be essentially induced by tuberculous infection, previous tuberculous infection might simply played a role to re-construct airway, and an active tuberculous infection has little contribution to develop the pulmonary lesion.

Some our CPA cases showed pre-existing airway remodeling, such as cavitary formation, most of which might be sequel to old tuberculosis followed by emphysema. This anatomical reconstruction can promote infestation or saprophytic proliferation of mold onto the 


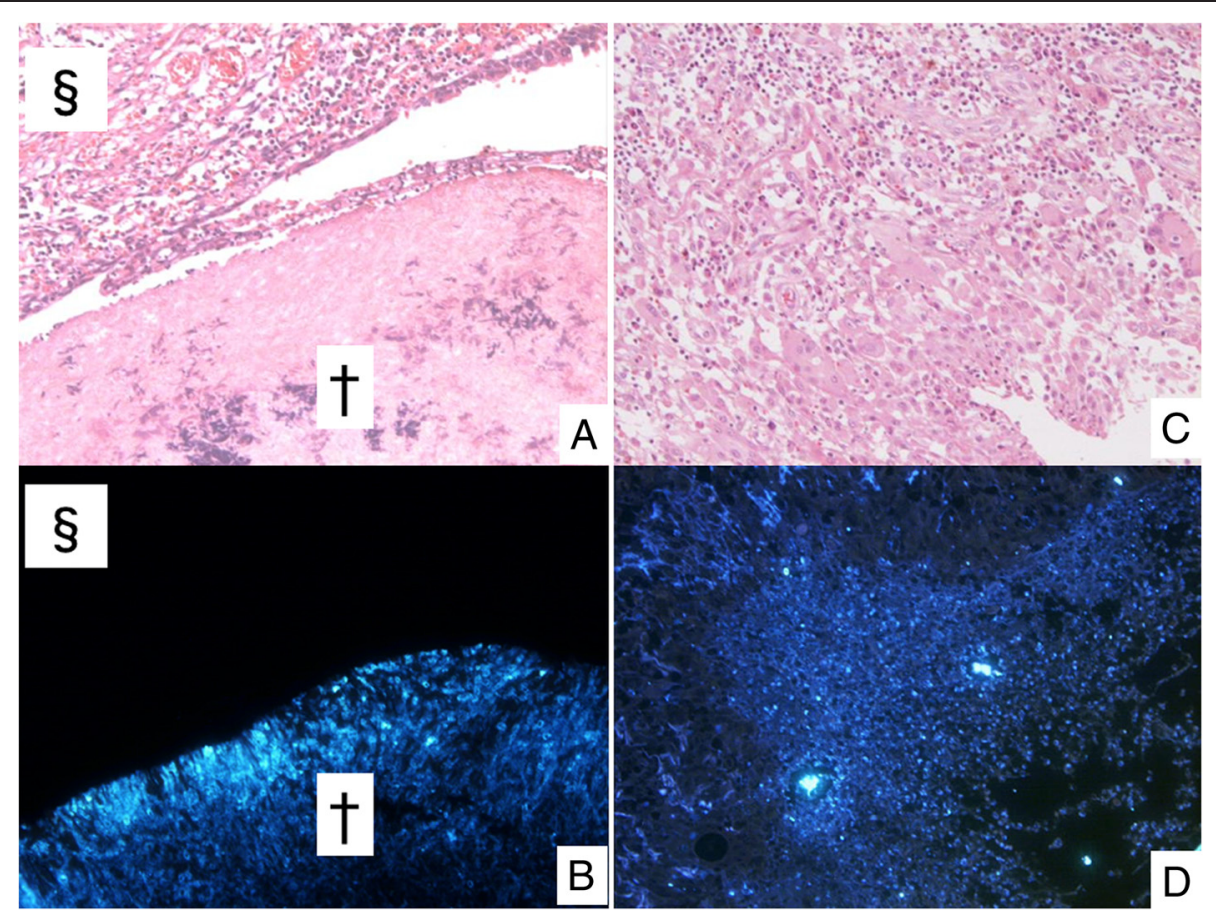

Fig. 4 a Fungus ball $(\dagger)$ and surrounded cavity $(\S)$ can be seen ( $\mathrm{H}-\mathrm{E}$ staining). b Positive signal can be detected only in fungus ball using Fungiflora $Y^{\ominus}$. $\mathbf{c}$ There is erosive area without fungal aspects ( $\mathrm{H}$-E staining). $\mathbf{d}$ Granular fluorescent signal at granulation tissue surrounded the cavity

lining epithelial cells of the altered airway. Whereas saprophytic proliferation of mold itself may irritate lining epithelial cells to occur erosion, mild impairment of the immune system indexed by steroid administration and/or diabetes mellitus in our study can play an important role to induce active state of erosion or ulcer accompanied with massive exudate. There was little hyphal component in inflammatory exudate filling the alveolar space even that has no direct connection to altered large air spaces containing molds. Therefore, organization associated with CPA usually led severe respiratory failure can be understood as result of spreading of acute inflammatory exudate provided from active erosion of the altered airway, mostly corresponding to cavity, but progression of organization was not concerned whether the exudate containing fungal element or not. Another analysis of our study revealed diffusion of fungal components at the organizing area around the cavity wall, which could be detected as fine fluorescent granules by Fungiflora $Y^{\ominus}$ system. Consequently, We wish to emphasize that the organizing pneumonia known as a serious complication of the CPA is induced by spreading of acute inflammatory exudate via airway, and this phenomena has much lesser concerning whether mold is viable or not. On the other hand, whereas $\mathrm{BD}$ testing has been accepted as one of the useful tool for diagnosis of invasive aspergillosis, of which value is reflecting extent of fungal exposure to the blood flow, implication of BD test has still been controversial in case of CPA. A part of this may be explained that mold intermingled with inflammatory exudate in the cavity is isolated from the patient's blood flow with various degree by the altered airway usually consisted with inflammatory granulation tissue and fibrous tissue including neutrophils, mononuclear cells, and fibroblasts. In the present study, we found a negative correlation between WBC and BD, although it was not statistically significant, which suggests that neutrophil activity not only kill the invading fungi, but also prevent to transfer BD from feeble fungi into blood flow, since the main defense against mold is the action of neutrophils. As described above, some CPA cases showed an organization area without a fungal body. In addition, we detected a granular fluorescent signal in inflammatory granulation tissue surrounding the cavity in 4 cases (16\%) without fungal aspects (GF). Among these cases, which had a measured BD before the surgical resection, we found a statistically significant association between a high level of $\mathrm{BD}$ and the presence of GF. Therefore, an increasing of BD in case of CPA can be understood as a sequel to widely spread diffusion of fungal components largely consisted with $\mathrm{BD}$. There may be much lesser contribution to increase BD that the direct penetration of fungi into the tissue or blood flow, of which situation is usually confirmed in case of invasive aspergillosis with agranulocytosis [7]. 


\section{Conclusions}

We wish to conclude that extension of acute inflammatory exudate along the terminal respiratory tract is most significant pathophysiological complication of the CPA, because the spreading of exudate filling alveolar space must cause organizing pneumonia, which derives fatal respiratory failure. In addition, the viability commonly suggesting the invasiveness of saprophytic Aspergilli does not concern progression of exudative inflammation at the site of erosion.

\section{Competing interests}

Dr. Shibuya reports receiving research grants from Pfizer Inc., Janssen Pharmaceutical K.K., Dainippon Sumitomo Pharma Co., Astellas Pharma Inc., Taiho Pharmaceutical Co., and POLA-Pharma Inc. Other authors declare that they have no competing interests.

\section{Authors' contributions}

All authors contributed towards the conceptualization, writing, reading, and approval of the final manuscript. In particular, NT and TI jointly conceptualized this study, integrated the data, wrote the manuscript, and contributed equally to this work.

\section{Acknowledgment}

This work was supported by Health Science Research Grants for Research on Emerging and Re-emerging Infectious Diseases (grant numbers: $\mathrm{H} 25-$ shinkouippan-006 and H26-shinkoujitsuyouka-ippan-010) from the Ministry of Health, Labour and Welfare of Japan, a grant from the Strategic Basis on Research Grounds for Non-governmental Schools at Heisei 20th, the Strategic Research Foundation Grant-aided Project for Private Schools at Heisei 23rd, KAKENHI (grant numbers: \#24790364, 26860250, 26460460, and 26860774) from the Ministry of Education, Culture, Sports, Science, and Technology of Japan, Toho University project grants (grant numbers: \#23-19, 21, and 28, and \#24-11, 16, and 28, and \#25-33), a Yokohama Foundation for the Advancement of Medical Science grant to YO, KA, and MS, Dr. Yanase's grant from Toho University Medical School to YO, and Kurozumi Medical Foundation grant to NT.

\section{Author details}

'Department of Surgical Pathology, Toho University School of Medicine, 6-11-1 Omori-nishi, Ota-ku, Tokyo 143-8541, Japan. ${ }^{2}$ Division of Respiratory Medicine, Toho University School of Medicine, 6-11-1 Omori-nishi, Ota-ku, Tokyo 143-8541, Japan. ${ }^{3}$ Division of Chest Surgery, Toho University School of Medicine, 6-11-1 Omori-nishi, Ota-ku, Tokyo 143-8541, Japan. ${ }^{4}$ Laboratory of Molecular Cell Biology, School of Pharmacy, Nihon University, 7-7-1

Narashinodai, Funabashi-shi, Chiba 274-8555, Japan.

Received: 8 January 2015 Accepted: 27 August 2015

Published online: 03 September 2015

\section{References}

1. Grahame-Clarke CN, Roberts CM, Empey DW. Chronic necrotizing pulmonary aspergillosis and pulmonary phycomycosis in cystic fibrosis. Respir Med. 1994:88:465-8.

2. Gefter WB, Weingrad TR, Epstein DM, Oches RH, Miller WT. "Semi-invasive" pulmonary aspergillosis: a new look at the spectrum of Aspergillus infections of the lung. Radiology. 1981;140:313-21.

3. Binder RE, Faling LJ, Pugatch RD, Masasaen C, Snider GL. Chronic necrotizing pulmonary aspergillosis: a discrete clinical entity. Medicine (Baltimore). 1982;61:109-24.

4. Denning DW, Riniotis K, Dobrashian R, Sambatakou H. Chronic cavitary and fibrosing pulmonary and pleural aspergillosis: case series, proposed nomenclature change, and review. Clin Infect Dis. 2003;37 Suppl 3:S265-80.

5. Yousem SA. The histological spectrum of chronic necrotizing forms of pulmonary aspergillosis. Hum Pathol. 1997;28:650-6.

6. Izumikawa K, Tashiro T, Tashiro M, Takazono T, Kosai K, Morinaga Y, et al. Pathogenesis and clinical features of chronic pulmonary aspergillosis - Is it possible to distinguish CNPA and CCPA clinically? J Infect Chemother. 2014:20:208-12.

7. Shibuya K, Takaoka M, Uchida K, Wakayama M, Yamaguchi H, Takahashi K, et al. Histopathology of experimental invasive pulmonary aspergillosis in rats: pathological comparison of pulmonary lesions induced by specific virulent factor deficient mutants. Microb Pathog. 1999;27:123-31.

\section{Submit your next manuscript to BioMed Central and take full advantage of:}

- Convenient online submission

- Thorough peer review

- No space constraints or color figure charges

- Immediate publication on acceptance

- Inclusion in PubMed, CAS, Scopus and Google Scholar

- Research which is freely available for redistribution 